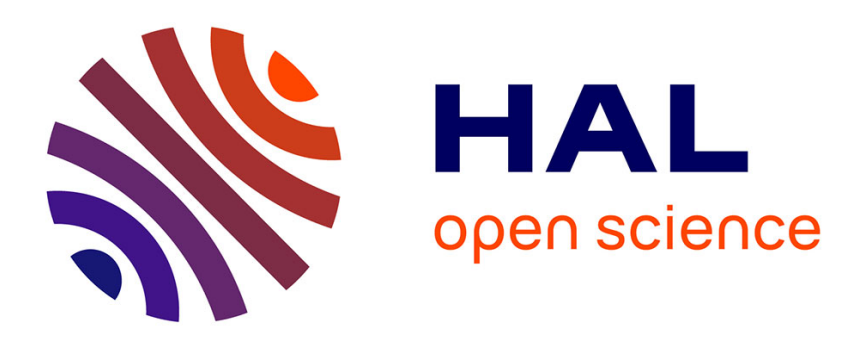

\title{
Origine structurale et chimique de l'activité électrique des joints de grains dans le silicium
}

\author{
A. Rocher
}

\section{To cite this version:}

A. Rocher. Origine structurale et chimique de l'activité électrique des joints de grains dans le silicium. Revue de Physique Appliquée, 1987, 22 (7), pp.591-595. 10.1051/rphysap:01987002207059100 . jpa00245581

\section{HAL Id: jpa-00245581 https://hal.science/jpa-00245581}

Submitted on 1 Jan 1987

HAL is a multi-disciplinary open access archive for the deposit and dissemination of scientific research documents, whether they are published or not. The documents may come from teaching and research institutions in France or abroad, or from public or private research centers.
L'archive ouverte pluridisciplinaire HAL, est destinée au dépôt et à la diffusion de documents scientifiques de niveau recherche, publiés ou non, émanant des établissements d'enseignement et de recherche français ou étrangers, des laboratoires publics ou privés. 
Classification

Physics Abstracts

$61.70 \mathrm{~N}-72.20 \mathrm{~J}$

\title{
Origine structurale et chimique de l'activité électrique des joints de grains dans le silicium
}

\author{
A. Rocher \\ Laboratoire d'Optique Electronique du CNRS, 29, rue Jeanne-Marvig, 31400 Toulouse, France
}

(Reçu le 26 mars 1987, accepté le 3 avril 1987)

\begin{abstract}
Résumé. - Cet article présente un bilan des études que nous avons menées sur les joints de grains dans le silicium par microscopie électronique en transmission et à balayage (SEM/EBIC). Le principal résultat est que la structure cristalline ne gouverne, en général, pas seule les propriétés électriques des joints. Un défaut cristallin agit d'abord sur les phénomènes de diffusion et de ségrégation d'impuretés. L'ensemble a alors ses propres propriétés électriques.
\end{abstract}

\begin{abstract}
This paper forms a final report on our studies on grain boundaries in silicon by electron microscope techniques TEM and SEM/EBIC. The main result is that the crystalline structure is not the main agent of the grain boundary electrical properties. A crystalline defect acts firstly on the diffusion and segregation of impurities. This entity has its own electrical properties.
\end{abstract}

\section{Introduction.}

Les études menées sur le silicium polycristallin destiné aux photopiles solaires avaient pour but initial de préciser le rôle des défauts et plus spécialement celui des joints de grains sur les rendements de conversion photovoltaïque. Compte tenu des progrès technologiques concernant la croissance cristalline et la purification des matériaux il était important de réactualiser et préciser les connaissances acquises sur ce sujet dans les années cinquante. Avec les tailles de grains intervenant dans la plupart des photopiles commercialisées, il est rapidement apparu que les joints de grains ne perturbaient par euxmêmes que faiblement les performances de conversion. Cependant les impuretés et les défauts cristallins liés aux modes de croissance sont à l'origine d'un rendement moins élevé que pour le silicium monocristallin. Si du point de vue des applications de nombreux problèmes ont été résolus, des questions fondamentales demeuraient encore sans réponse. En particulier, quel type de relation existe entre la structure cristalline et les propriétés électriques d'un défaut ? Deux techniques de microscopie électronique ont été utilisées pour étudier les joints de grains dans le silicium et répondre ainsi à cette question : 1) le mode $S E M / E B I C$ qui permet de mettre en évidence les zones de forte recombinaison des porteurs de charge au niveau des joints, 2) la microscopie électronique en transmission qui en caractérise la structure cristalline. La mise en évidence par contraste EBIC d'un comportement électrique particulier est apparue comme un critère judicieux pour déterminer si la structure d'un joint devait être ou non étudiée par TEM [1-4].

\section{Techniques expérimentales.}

Les échantillons étudiés proviennent aussi bien de matériaux polycristallins destinés aux applications solaires (lingots SILSO ou rubans RAD) que de bicristaux artificiels de silicium.

Les photopiles de bonne qualité sont principalement obtenues avec des matériaux peu contraints où les grains présentent entre eux des relations d'orientation mutuelle dites de coïncidence. Ces relations, étudiées par diffraction électronique, sont souvent caractérisées par des rotations d'axe $\langle 110\rangle$ et d'angles multiples de $70^{\circ} 53$. Cette multiplicité correspond à différents ordres de macle qui ont été trouvés compris entre 1 et 5 [5]. De plus, les interfaces ont tendance à suivre le gradient de température et leur géométrie est en relation directe avec la forme du front de croissance. Ils ne sont en général pas plans 
sur de grandes surfaces. Ils peuvent être quelconques ou constitués de marches composées des plans miroirs associés à la relation de macle du joint.

Les bicristaux étudiés sont obtenus par tirage Czochralski à partir de deux germes convenablement orientés pour fournir des joints dont les caractéristiques d'orientation et de plan d'interface ont été choisies a priori [6]. Bien définis cristallographiquement sur de grandes dimensions, ils permettent des études systématiques sur plusieurs paramètres. De plus, leurs comportements électriques sont bien identifiables.

La technique SEM/EBIC ne sera pas discutée ici, car elle a déjà fait l'objet de présentations complètes $[3,7]$. Elle est bien adaptée à la caractérisation des défauts dans les photopiles car basée exactement sur le même principe que la conversion photovoltaïque : un rayonnement extérieur (le faisceau d'électrons remplace le soleil) crée des paires électron-trou, qui sont séparées et collectées par une jonction p-n ou une diode Schottky. Ces expériences, généralement réalisées à $30 \mathrm{kV}$ dans des conditions de faible injection, permettent de dresser avec une résolution de quelques microns une carte de densité des centres de recombinaison des porteurs de charge. Ces centres de recombinaisons se trouvent le plus souvent localisés au voisinage des défauts étendus tels que dislocations, joints de grains ou précipités.

Les études de microscopie électronique en transmission nécessitent des épaisseurs d'échantillon qui doivent être, selon la tension utilisée et la résolution demandée, comprises entre quelques nanomètres et quelques microns. Pour cela, les échantillons sont amenés mécaniquement jusqu'à des épaisseurs de l'ordre de 100 microns. Puis après repérage par photo-optique, les régions présentant des contrastes intéressant en EBIC sont amincies par usinage ionique [3]. Les observations sont faites à des tensions comprises entre 100 et $2000 \mathrm{kV}$.

\section{Origine cristalline de l'activité électrique des joints de grains.}

Une grande multiplicité de structure de joints et donc de comportements électriques sont observés dans les matériaux polycristallins destinés aux applications solaires (SILSO ou RAD). Nous avons cherché à relier l'origine d'un comportement électrique particulier mis en évidence par contraste EBIC à une structure cristallographique spécifique. Deux cas sont discutés :

2.1 VARIATION DU CONTRASTE EBIC AVEC LA STRUCTURE CRISTALLINE D'INTERFACE. - Le premier échantillon a été prélevé au centre d'un lingot SILSO. Il a été recuit en atmosphère humide à $1150{ }^{\circ} \mathrm{C}$ pour réaliser un oxyde de protection en surface où, après ouverture de l'oxyde par attaque chimique, une diode Schottky circulaire au chrome a été déposée.

L'image EBIC présentée sur la figure 1a montre un changement abrupt du pouvoir de recombinaison d'un joint de grains : la partie (I1) a une très forte activité électrique qui s'arrête de façon abrupte. Or un joint est une surface fermée qui ne peut se terminer que sur un autre joint ou en surface. La partie notée (I2) existe donc bien mais ne présente aucune activité électrique [3].
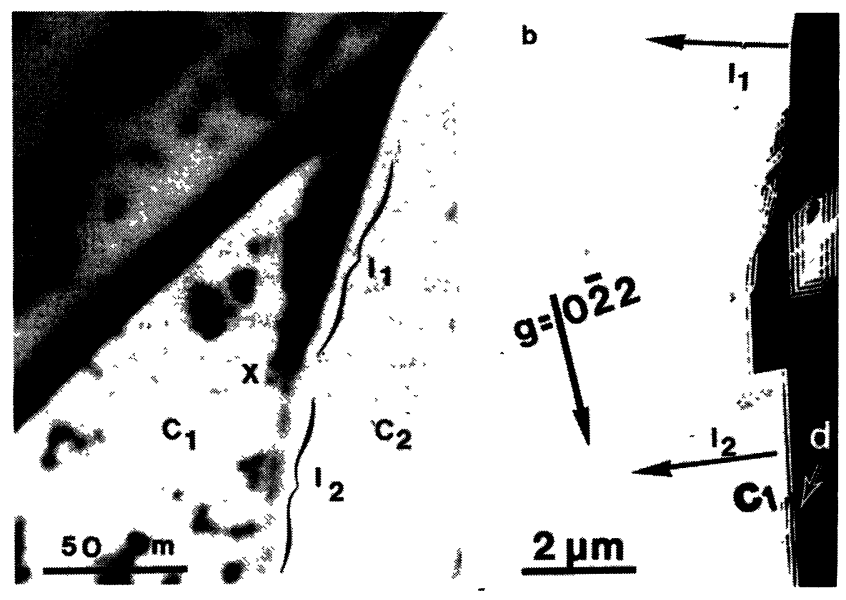

Fig. 1. - a) Image EBIC à $30 \mathrm{kV}$. Le joint de grains indexé $I$ a deux activités électriques très différentes mises en évidence par des contrastes EBIC fort en I1 et faible en I2. b) Micrographie prise à $2 \mathrm{MeV}$ en champ sombre 022 . L'interface I1 est incohérent. Il a une normale proche de (111) dans un grain et de (110) dans l'autre. L'interface I2 est un plan miroir (221), contenant des dislocations intergranulaires $\mathrm{d}$.

[a) $30 \mathrm{kV}$ EBIC image. The grain boundary indexed I presents two different electrical activities shown by strong and weak EBIC contrast for I1 and I2. b) $2 \mathrm{MeV}$ TEM micrographs taken in dark field. The interface $I 1$ is incoherent. It is characterized by a normal close to (111) in one grain and to (110) in the other. The interface $I 2$ is a mirror plane (221), containing the intergranular dislocations d.]

L'origine de ce changement a été établie grâce à des observations en TEM à $2 \mathrm{MeV}$. La figure $1 \mathrm{~b}$, réalisée en champ sombre avec le plan réflecteur 220 d'un des grains, montre la région de changement de contraste EBIC. De part et d'autre du joint on observe deux grains dont la relation d'orientation mutuelle a été définie comme une macle du second ordre $(\Sigma=9)$. Deux configurations d'interface différentes sont observées [13].

La portion d'interface I2, qui ne présente aucune activité électrique, a été indexée comme un plan miroir $(221) /(221)$. La structure cristalline de cette interface a la périodicité du réseau de coïncidence. 
Elle est constituée de deux motifs de dislocation de Lomer qui assurent avec une distorsion raisonnable la tétracoordinance des liaisons ne donnant lieu à aucun niveau d'énergie dans la bande interdite [810]. Le joint n'a donc aucune activité électrique notable.

L'autre portion, recombinante, n'est pas strictement plane. Elle a été indexée proche de (110)/(111), qui ne correspond donc pas à un plan miroir. Cette interface n'a, a priori, aucune structure périodique qui lui permette de minimiser le nombre de liaisons perturbées voire pendantes. Elle présente une activité électrique importante.

L'origine de l'activité électrique est ici directement reliée à un effet cristallographique. Cependant il n'est pas possible de déterminer à partir de ces observations quel est le rôle respectif des liaisons déformées et des phénomènes d'impuretés reliés aux contraintes associées au défaut.

2.2 DisLOCATIONS DANS LES JOINTS. - Les dislocations intergranulaires peuvent jouer un rôle sur l'activité électrique des joints. Ce rôle dépend de leurs natures cristallines. Dans le cas précédent, il existe dans l'interface $\mathrm{I} 2$ des dislocations rectilignes, repérées par d sur la figure 1b. Ces dislocations, dont la densité est d'environ une par micron, sont des dislocations à $60^{\circ}$ portées par la direction $\langle 110\rangle$ commune aux deux grains. Elles ne donnent lieu à aucun contraste EBIC observable et n'ont donc pas d'activité électrique importante à température ambiante.

La figure $2 a$ montre une micrographie EBIC sur laquelle on observe de nombreux contrastes de joints. Le contraste noté M1 est apparu intéressant car il est rectiligne et important. La figure $2 b$ est une micrographie réalisée à $2 \mathrm{MeV}$ qui montre le défaut responsable de ce contraste EBIC : une micromacle dont les plans d'interface contiennent une grande densité de dislocations dissociées. Ces dislocations courbes induisent une très forte activité électrique. Ce résultat a été également observé par Gleichmann et al. qui discutent principalement du rôle d'obstacle que le joint de macle peut jouer sur le glissement et la dissociation des dislocations [11].

\section{Origine chimique de l'activité électrique des joints de grains.}

Dans le cas des photopiles les traitements thermiques sont nécessaires pour réaliser la structure de collecte des porteurs de charge qui demeure la traditionnelle jonction pn réalisée par diffusion à des températures de l'ordre de $800^{\circ} \mathrm{C}$. Ces traitements améliorent aussi la qualité électronique du matériau. Ainsi, un recuit de $2 \mathrm{~h}$ à $450^{\circ} \mathrm{C}$ sous azote permet de doubler la longueur de diffusion des porteurs minoritaires [12].

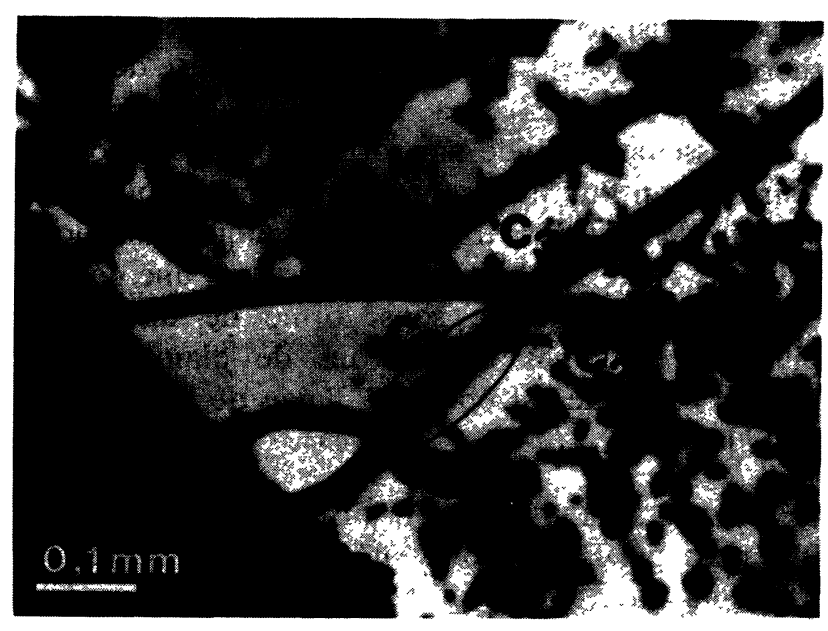

a)

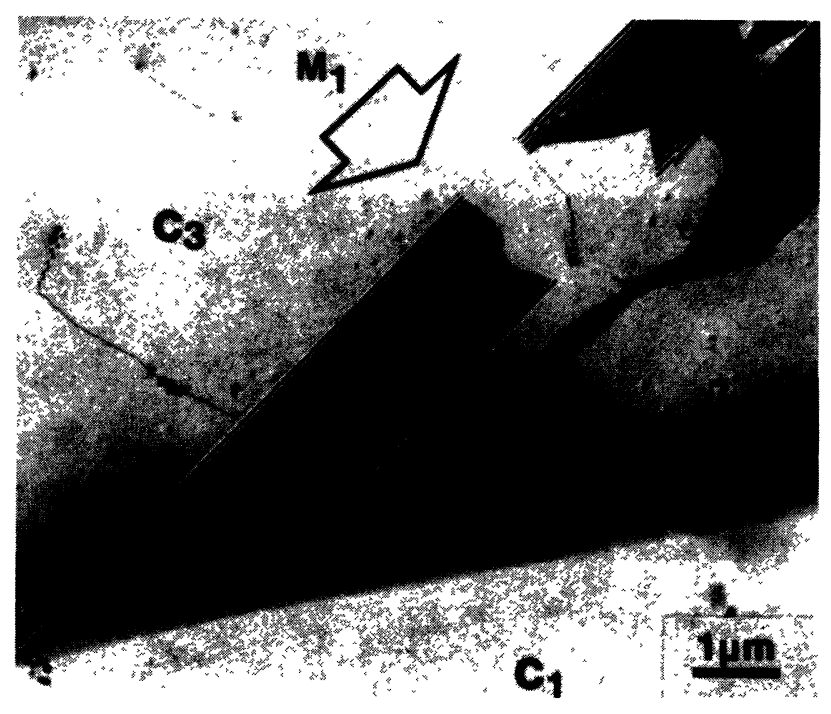

b)

Fig. 2. - a) Image EBIC à $30 \mathrm{kV}$. b) Champ clair à $2 \mathrm{MeV}:$ le contraste EBIC rectiligne noté M1 est associé à la micromacle M1 qui contient une forte densité de dislocations courbes dans ses plans d'interface.

(a) $30 \mathrm{kV}$ EBIC image. b) $2 \mathrm{MeV}$ bright field: The straight EBIC contrast, labelled M1, is due to the microtwin M1, which contains a high density of curved dislocations in its interfaces.]

Les propriétés électriques du silicium et des défauts qui lui sont associés dépendent très fortement de leurs histoires thermiques. Bruts de croissance, les lingots de silicium fournissent des joints de grains sans activité électrique $[3,13]$. Pourtant les structures de joints ne sont pas simples et devraient présenter une activité électrique importante si les effets cristallins n'étaient pas compensés par des impuretés. Des traitements thermiques exploratoires ont été effectués sur des bicristaux pour mettre en évidence les mécanismes qui intervenaient dans le changement d'activité électrique des joints de grains. 
Les bicristaux de silicium ont subi le traitement thermique suivant : montée en température de $10{ }^{\circ} \mathrm{C}$ par min, 2 ou $24 \mathrm{~h}$ à $750^{\circ} \mathrm{C}$ sous azote, puis refroidissement rapide par sortie de l'échantillon du four sous un flux intense d'azote. Après avoir retiré l'oxyde de surface par attaque chimique une diode Schottky a été réalisée. Elle consiste en une couche de $30 \mathrm{~nm}$ de chrome sous forme de disque de plusieurs centaines de microns de diamètre. Le contact ohmique est obtenu à l'aide de l'eutectique AuSi [12].

3.1 ACtivité ÉlectriQue discontinue. - Les observations EBIC, présentées sur la figure 3a, montrent que, pour les échantillons recuits pendant $2 \mathrm{~h}$, l'activité électrique au niveau du joint est discontinue : de grandes parties non actives électriquement sont séparées par de forts contrastes EBIC ponctuels.
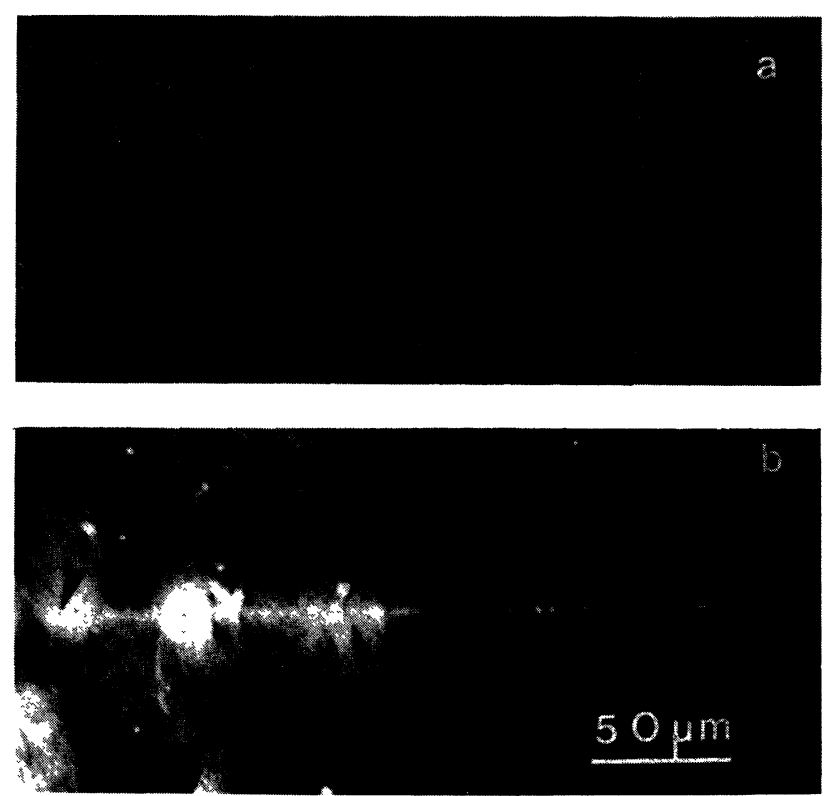

Fig. 3. - a) Image EBIC d'un bicristal de silicium $\Sigma 9$, recuit 2 heures à $750^{\circ} \mathrm{C}$. Un contraste EBIC hétérogène est observé le long du joint. Les taches noires sont reliées à des zones de fortes densités de centres de recombinaison. b) Image optique après révélation chimique. Des figures d'attaques reliées à des précipités sont révélées au joint. Une corrélation directe peut être établie entre les contrastes ponctuels EBIC et les figures d'attaques (1 à 12).

(a) EBIC image of a $\Sigma 9$ silicon bicrystals after 2 hours of heat treatments at $750^{\circ} \mathrm{C}$. Inhomogeneous EBIC contrast is observed along the grain boundary. The black dots are related to high density of recombination centres. b) Optical image after chemical etching. Etch pits related to precipitates are revealed at the grain boundary. A direct correlation can be established between EBIC black dots and precipitates (1 to 12$)$.]
La photographie faite par microscopie optique de la figure $3 \mathrm{~b}$ montre le résultat d'une révélation chimique sur l'échantillon étudié en EBIC: elle révèle au niveau du joint une succession de figures d'attaque qui correspondent exactement aux régions actives électriquement. De telles figures sont directement reliées à la présence de précipités qui se sont développés durant les traitements thermiques au niveau du joint. Leur répartition est hétérogène dans le joint. Des portions de joint sont propres, d'autres contiennent une forte densité de ces précipités. Ces fortes densités se trouvent dans des régions de concentration élevée d'oxygène qui sont directement mises en évidence par topographie $X$ sous forme de striation [14]. Ces précipités, souvent en forme de galette d'un à deux microns de diamètre et de quelques nanomètres d'épaisseur, introduisent des champs de contrainte sur quelques microns. Ils ne paraissent pas uniquement constitués d'oxygène. En effet, compte tenu de sa concentration moyenne dans le matériau, l'oxygène ne semble pas assez mobile pour expliquer les tailles de précipités atteintes après deux heures à $750^{\circ} \mathrm{C}$ [14]. Des analyses préliminaires montreraient que ces précipités ne contiennent pas uniquement de l'oxygène mais qu'ils sont constitués d'atomes plus lourds [15]. Il pourrait s'agir d'une contamination par le cuivre, très mobile à ces températures. Cette pollution apparaîtrait lors des différents stades de préparation et de nettoyage des échantillons ou durant leurs traitements thermiques.

3.2 RECOMBINAISON HOMOGÈNE AUX JOINTS. Avec des traitements thermiques de $24 \mathrm{~h}$ à $750^{\circ} \mathrm{C}$ l'activité électrique des joints devient, en bonne approximation, uniforme. Les vitesses de recombinaison ont été mesurées pour différentes structures de bicristaux ayant subi les mêmes traitements thermiques [16]. Celle du joint (221) $\Sigma 9$ a été trouvée environ deux fois plus faible que celles associées aux joints (510) $\Sigma 13$ et (710) $\Sigma 25$ [16]. Cette différence a pour origine probable des relations d'orientation qui sont, selon les cas, exactes ou non. Ainsi le bicristal $\Sigma 9$, obtenu à partir d'un germe naturel, a une relation de coïncidence parfaite. Au contraire les deux bicristaux $\Sigma 13$ et $\Sigma 25$, réalisés à partir de germes orientés artificiellement, présentent un écart à la coïncidence, qui est rattrapé par un réseau de dislocations primaires. La figure 4 montre un tel réseau de dislocations pour le bicristal $\Sigma 13$. Les dislocations qui le composent sont décorées et distantes de $25 \mathrm{~nm}$. Cette décoration montre le rôle important qu'elles peuvent jouer sur les phénomènes de ségrégation et de diffusion d'impuretés. Elles ont tout lieu d'être responsables de l'activité électrique de ce joint qui est deux fois supérieure à celle d'un joint en coïncidence exacte. 


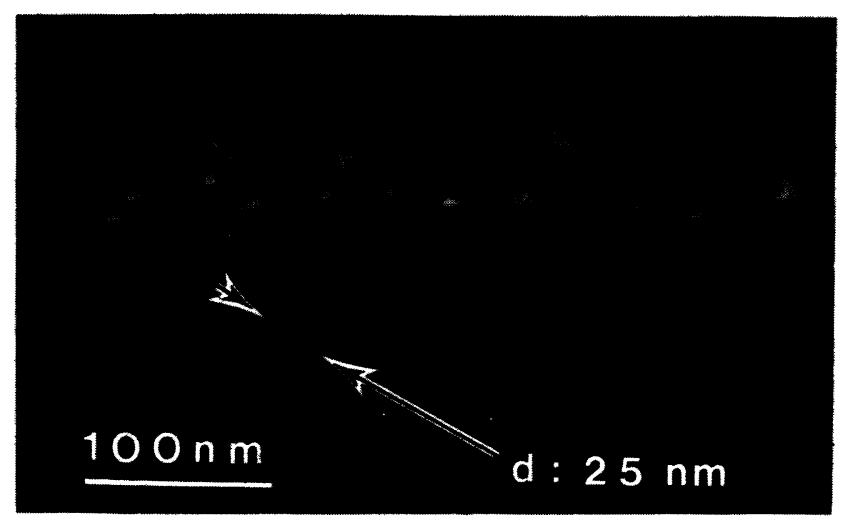

Fig. 4. - Image en fond noir réalisée à $200 \mathrm{kV}$ : réseau de dislocations décorées qui est responsable de la forte activité électrique du joint (510) $\Sigma 13$ après un traitement thermique de 24 heures à $750^{\circ} \mathrm{C}$.

[200 kV dark field image : network of decorated dislocations, which is responsible for the strong electrical activity of the (510) $\Sigma 13$ grain boundary after a 24 -hour heat treatment at $750{ }^{\circ} \mathrm{C}$.]

\section{Conclusion.}

Une relation directe a pu être établie entre l'activité électrique et la structure cristalline d'un joint de grains dans le silicium. L'absence d'activité électrique intrinsèque n'est observée que pour des configurations très particulières de joint correspondant à des structures reconstruites telles que celles obtenues pour les plans miroirs (111) $\Sigma 3$ ou (211) $\Sigma 9$. La perte du plan miroir est suffisante pour rendre à un joint ayant une relation de coïncidence son caractère général et donc lui associer la possibilité d'une activité électrique importante.

Ces études conduisent à penser que l'activité électrique des joints dans le silicium ne dépend pas essentiellement de sa structure cristalline mais plutôt de son environnement chimique. Dans les conditions actuelles de pureté des matériaux, il ne paraît pas concevable d'avoir un joint macroscopique pour lequel il soit possible d'isoler un comportement électrique uniquement lié à un effet cristallin. La structure cristalline du joint agit d'abord sur les phénomènes de ségrégation d'impuretés. L'ensemble a ensuite des propriétés électriques globales propres. Ainsi l'utilisation de traitements thermiques en atmosphère contrôlée permet de modifier l'activité électrique en l'augmentant ou en la diminuant. Des études plus systématiques et approfondies devraient conduire à une meilleure compréhension des phénomènes d'activation et de passivation des défauts de structures dans le silicium.

\section{Remerciements.}

Cet article a été rédigé d'après les thèses de Chantal Fontaine, Christiane Dianteill et Florent Battistella. Qu'ils soient remerciés ici de leur collaboration. Je voudrais aussi remercier Christian Belouet (CGE, LDM) pour les discussions que nous avons eues pour orienter ces travaux. Ces études se sont développées grâce au soutien financier du PIRSEM et de l'AFME au travers de l'ARC « silicium polycristallin ».

\section{Bibliographie}

[1] Texier, C., Thèse $3^{\mathrm{e}}$ Cycle (1980) Paris VII ; Sharko, R., Gervais, A. et Texier-Hervo, C., $J$. Physique Colloq. 43 (1982) C1-129.

[2] Cunningham, B., Strunk, H. P. and Ast, D. G., Materials Research Society Symposium Proc. (Elsevier, New York) 1982, vol. 5, p. 51.

[3] Dianteill, C., Thèse de docteur ingénieur (1983) Toulouse ;

Dianteill, C. et Rocher, A., J. Physique Colloq. 43 (1982) C1-75.

[4] Ruterana, P., Bary, A. et Nouet, G., J. Physique Colloq. 43 (1982) C1-27.

[5] Fontaine, C., Thèse de docteur ingénieur (1980) Toulouse; Fontaine, C. et Rocher A., J. Microsc. 118 (1980) 105.

[6] Aubert, J. J. and Bacmann, J. J., ce numéro.

[7] Leamy, H. J., J. Appl. Phys. 53 (1982) R51.

[8] Bourret, A., D'Anterroches, C. et Penisson, J. M., J. Physique Colloq. 43 (1982) C6 83.
[9] LANNOO, M., Les joints de grains dans les matériaux (Editions de Physique, les Ulis) 1985, p. 123.

[10] Thomson, R. E. and Chadi, D. J., Phys. Rev. B 29 (1984) 889.

[11] Gleichmann, R., Vaudin, M. D. and Ast, D. G., Philos. Mag. A 51 (1985) 449.

[12] Battistella, F., Thèse de docteur ingénieur (1985) Toulouse ;

Battistella, F. et Rocher, A., Proc. of the 6th European Photovoltaic solar Energy conference, Londres (1985) p. 113.

[13] Kazmerski, L. L. and Russel, P. E., J. Physique Colloq. 43 (1982) C1-171.

[14] Battistella, F., Rocher, A. et George, A., Mat. Res. Soc. Proc. (Elsevier, New York) 59 (1986) 347.

[15] Battistella, F., communication privée.

[16] BAttistella, F. et Rocher, A., à paraître dans Semiconductor Science and Technology (1987). 